\title{
Inferring Ideal Amino Acid Interaction Forms From Statistical Protein Contact Potentials
}

\author{
Piotr Pokarowski, ${ }^{1 *}$ Andrzej Kloczkowski, ${ }^{2}$ Robert L. Jernigan, ${ }^{2}$ Neha S. Kothari, ${ }^{2}$ Maria Pokarowska, ${ }^{3}$ and \\ Andrzej Kolinski ${ }^{4}$ \\ ${ }^{1}$ Institute of Applied Mathematics and Mechanics, Warsaw University, Warsaw, Poland \\ ${ }^{2}$ Laurence H. Baker Center for Bioinformatics and Biological Statistics, Iowa State University, Ames, Iowa \\ ${ }^{3}$ Faculty of Geodesy and Cartography, Warsaw University of Technology, Warsaw, Poland \\ ${ }^{4}$ Laboratory of Theory of Biopolymers, Faculty of Chemistry, Warsaw University, Warsaw, Poland
}

\begin{abstract}
We have analyzed 29 different published matrices of protein pairwise contact potentials (CPs) between amino acids derived from different sets of proteins, either crystallographic structures taken from the Protein Data Bank (PDB) or computergenerated decoys. Each of the CPs is similar to 1 of the 2 matrices derived in the work of Miyazawa and Jernigan (Proteins 1999;34:49-68). The CP matrices of the first class can be approximated with a correlation of order 0.9 by the formula $e_{i j}=h_{i}+h_{j}, 1 \leq i, j \leq 20$, where the residue-type dependent factor $h$ is highly correlated with the frequency of occurrence of a given amino acid type inside proteins. Electrostatic interactions for the potentials of this class are almost negligible. In the potentials belonging to this class, the major contribution to the potentials is the one-body transfer energy of the amino acid from water to the protein environment. Potentials belonging to the second class can be approximated with a correlation of 0.9 by the formula $e_{i j}=c_{0}-h_{i} h_{j}+q_{i} q_{j}$, where $c_{0}$ is a constant, $h$ is highly correlated with the Kyte-Doolittle hydrophobicity scale, and a new, less dominant, residue-type dependent factor $q$ is correlated $(\sim 0.9)$ with amino acid isoelectric points pI. Including electrostatic interactions significantly improves the approximation for this class of potentials. While, the high correlation between potentials of the first class and the hydrophobic transfer energies is well known, the fact that this approximation can work well also for the second class of potentials is a new finding. We interpret potentials of this class as representing energies of contact of amino acid pairs within an average protein environment. Proteins 2005;59:49-57. ๑) 2005 Wiley-Liss, Inc.
\end{abstract}

Key words: protein folding; protein structure prediction; threading; residue-based contact potentials; statistical potentials; knowledge-based potentials

\section{INTRODUCTION}

Statistical pairwise contact potentials $(\mathrm{CPs})$ of protein residues, have been derived either by using the quasichemical approximation from databases of proteins having known structures, ${ }^{1-23}$ or by fitting their values to optimize the selection of the correct structures as the lowest energy forms in comparisons against sets of misfolded structures (decoys). ${ }^{24-30} \mathrm{CPs}$ have been increasingly heavily used over the last 20 years for ligand docking, fold recognition, and protein structure prediction from amino acid sequence (see review papers ${ }^{31-33}$ ). Analysis of results of the Critical Assessment of Techniques for Protein Structure Prediction (CASP) experiment shows that most of the successful groups use statistical CPs in their force fields for threading or ab initio protein structure prediction. ${ }^{34-41}$

Introducing the same level of coarse graining over structures, as is considered in protein sequences, has a major advantage for relating sequences to structures. It is well known that coarse graining of structures removes some of the specificity. For example, when the level of structural representation is 1 point per amino acid, then some of the details of backbone conformation are lost, but most of the information regarding side conformations is thrown away. Successful use of CPs coarse grained at this level relies upon the underlying assumption that the terms coming from the atomic details will be less important than the placement of the residues within the structure overall. While there has been no rigorous proof of this, there is now a large body of evidence in support of this view coming from the widespread use of the CPs.

In the present work, we first compare 29 different CPs currently used in computational biology. Each of these potentials is similar to one of the 2 matrices defined by Miyazawa and Jernigan. ${ }^{22}$ We then show that the actual contribution of specific two-body interactions to CPs is quite insignificant. The issue regarding higher body terms, of course, remains open. ${ }^{42}$ Nonetheless, all the known pairwise matrices of CPs can be surprisingly well approximated by simple functions of individual residue properties, such as hydrophobicity and electrostatic properties (in $\mathrm{pH}$ units given as isoelectric points pI), ${ }^{43-47}$ for each pair of amino acids. We term such an approximation of the CP matrices a one-body approximation. Hydrophobicity repre-

Grant sponsor: Polish Research Council KBN; Grant number: PBZ-KBN-088/P04/2003 (to A. Kolinski).

*Correspondence to: Piotr Pokarowski, Institute of Applied Mathematics and Mechanics, Warsaw University, Banacha 2, 02-097 Warsaw, Poland. E-mail: pokar@mimuw.ed.pl

Received 1 July 2004; Accepted 3 October 2004

Published online 1 February 2005 in Wiley InterScience (www.interscience.wiley.com). DOI: 10.1002/prot.20380 
sents the dominant factor in protein potentials, with other, less important factors being the energy of demixing of amino acids in a protein environment and electrostatic interactions. As we will see, the accuracy of the one-body approximation works significantly better for potentials derived from the quasi-chemical principle than for the potentials obtained from the optimization of the prediction of the native structures among decoys. This calls into question the quality of the decoys in general compared to the known structures. It is quite interesting that the frequencies of contacts between different amino acids can also be successfully approximated with the present method. Thus, hydrophobicity, demixing, and electrostatics are identifiable as fundamental properties defining potentials from the simple statistics of inter-residue pair contacts for proteins in the Protein Data Bank (PDB). Furthermore, an appropriate function form for combining these terms is obtained in the present work.

The one-body approximation helps us to comprehend the separation of the CPs into 2 classes. Potentials belonging to the first class are dominated by the one-body energies of transfer of amino acids from water to a protein environment. The matrices $\left(e_{i j}\right), 1 \leq i, j \leq 20$ representing this class of CPs can be approximated with the formula $e_{i j}=h_{i}$ $+h_{j}$, where the residue-type dependent coefficient $h$ strongly correlates with the frequency of occurrence of a given amino acid type inside proteins. Potentials belonging to the second class represent mostly energies of contacts of amino acids in a protein environment. The second class of potentials can be approximated with a correlation of order 0.9 by the formula $e_{i j}=c_{0}-h_{i} h_{j}+q_{i} q_{j}$. Here residue-type dependent factors $h$ and $q$ are highly correlated (both with a correlation of order 0.9 ) with the Kyte-Doolittle hydrophobicity scale and electrostatic property $\mathrm{pI}$, respectively, and $c_{0}$ is a constant. The electrostatic properties of an amino acid are represented by its isoelectric point and measured in $\mathrm{pH}$ units. The electrostatic interactions are quite important for the second class of potentials but are completely negligible for the first class of potentials.

The high correlation between potentials of the first class and transfer energies is well known. It seems somewhat surprising that the one-body approximation works well also for the second class of potentials, because these potentials have frequently been derived by excluding hydrophobic interactions. It can be shown that the term $c_{0}-h_{i} h_{j}$ (including hydrophobicity and energy of demixing) describes the dominant property of amino acid interactions in the protein environment leading to attraction between hydrophobic/polar like-type residues and repulsion between unlike-type residues that gives the spatial segregation between a protein's hydrophobic interior and polar surface. It is interesting that similar long-range interactions come from our minimal model of protein folding. ${ }^{48,49}$

The explicit inclusion of hydrophobic and electrostatic interactions allows us to analyze and compare various statistical potentials for proteins. On the other hand, a statistical analysis of the frequencies of pairwise residue contacts in protein structures leads to the derivation of explicit forms, which can be correlated and compared against various experimental scales of hydrophobicity and pI. Validating the potential form in this way permits us to comprehend these complex interactions.

\section{METHODS}

Because of the symmetry, we identify the matrix $E=$ $\left(e_{i j}\right)$ of contact potentials with its upper diagonal part $\left(e_{i j}\right)_{i \leq j}$. Our aim is to find a simple function $\tilde{E}(h, q)=\left[\tilde{e}(h, q)_{i j}\right]$ of two 20-dimensional vectors $h$ and $q$ (properties of the 20 amino acids) that minimizes the sum of squares

$$
\sum_{i, j: i \leq j}\left[e_{i, j}-\tilde{e}(h, q)_{i, j}\right]^{2} \rightarrow \min _{h, q}
$$

This defines the well known least squares problem.

Accuracy of the approximation is measured by the correlation coefficient, the relative Euclidean distance and the mean Euclidean distance between normalized matrices $E$ and $\tilde{E}$. Specifically, let us denote the scalar product of vectors $x$ and $y$ as $\langle x, y\rangle$ and the norm of $x$ as $\|x\|$ $=\sqrt{\langle x, x\rangle}$. The normalization of $x$ is given by the vector $x^{N}=(x-\bar{x}) / \sigma_{x}$, where $\bar{x}$ is the mean value of $x$ and $\sigma_{x}$ is its standard deviation, respectively. The correlation between vectors $x$ and $y$ is defined as

$$
\operatorname{cor}(x, y)=\frac{<x-\bar{x}, y-\bar{y}\rangle}{n \sigma_{x} \sigma_{y}}=\frac{\langle x-\bar{x}, y-\bar{y}\rangle}{\|x-\bar{x}\|\|y-\bar{y}\|} .
$$

We define also the distance between normalized vectors $\operatorname{dist}(x, y)=\left\|y^{N}-x^{N}\right\| / \sqrt{n}$, where $n$ is the dimensionality of vectors $x$ and $y$ (210 in our case). Obviously $\operatorname{dist}(x, y)$ is simply the root-mean-square difference between $y^{N}$ and $x^{N}$. In numerical analysis, it is popular to define the relative error of approximation of the vector $y$ by $x$ as $\operatorname{err}(x$, $y)=\|y-x\| /\|y\|$.

It is worth noting that all of the above defined measures of the quality of approximation (cor, dist, err) are invariant to multiplication by a scalar and are optimized by the solution of the least squares problem (Eq. 1). ${ }^{50}$ Additionally it is easily seen that $\operatorname{dist}^{2}(x, y)=2-2 \operatorname{cor}(x, y)$.

To approximate $E$, we investigate the following 4 simple functions:

$$
\begin{gathered}
\tilde{e}(h)_{i, j}=h_{i}+h_{j}(\mathrm{Hp}) \\
\tilde{e}(h)_{i, j}=c_{0}+c_{1} h_{i} h_{j}(\mathrm{Hp} \cdot \mathrm{Dx}) \\
\tilde{e}(h, q)_{i, j}=h_{i}+h_{j}+c_{0} q_{i} q_{j}(\mathrm{Hp} \cdot \mathrm{pH}) \\
\tilde{e}(h, q)_{i, j}=c_{0}+c_{1} h_{i} h_{j}+c_{2} q_{i} q_{j}(\mathrm{Hp} . \mathrm{Dx} \cdot \mathrm{pH}) .
\end{gathered}
$$

The above approximations are related to each other as follows:

$$
(\mathrm{Hp}) \rightarrow(\text { Hp.Dx }) \rightarrow(\text { Hp.pH }) \rightarrow(\text { Hp.Dx.pH })
$$

The relation (a) $\rightarrow$ (b) means that formula (b) is more general than (a); the proof is given in the Appendix. The 
simplest additive approximation is given by the vector $h$, which is often highly correlated with empirical hydrophobicities. Thus, we denote this approximation [Eq. 3(a)] by $(\mathrm{Hp})$. The solution of the least squares problem for this case [Eq. 3(a)] leads to linear equations that can be solved analytically:

$$
h_{i}=\left(s_{i}-c_{0}\right) /(n+2)
$$

with $n=20, s_{i}=\sum_{j} e_{i, j}+e_{i i}$ and $c_{0}=\left(\sum_{i j: i \leq j} e_{i, j}\right) /(n+1)$. All the other approximations given by Eqs. (3b)-(3d) lead to nonlinear least squares problems and require numerical solutions. We used the free software R (www.r-project.org) and Matlab with optimization toolbox (Mathworks, Inc; www.mathworks.com) in our computations. Four vectors were used as a starting solution for vector $h$ : the vector defined by Eq. (5), the diagonal $\left(e_{i i}\right)$, the eigenvectors of $E$, and the centered $E$ (matrix obtained from $E$ by subtracting the mean value) corresponding to the dominant eigenvalues. As the starting solution for $q$ we used isoelectric points designated here by $\mathrm{pH}$. To check the dependence of solutions of Eq. (3c) on the starting points, we interchanged vectors $h$ and $q$. This optimization is denoted as (pH.Hp). Generally, there is no significant dependence on starting vectors or the software used. The only exceptions were for MJ3h, TEl, B4, B5, and MSBM. On the other hand, for many CPs, the differences between solutions for (Hp.pH) and (pH.Hp) are essential.

Let us note that Eq. (3b) can be written in the following form:

$$
\tilde{e}(h, q)_{i, j}=h^{\prime}{ }_{i}+h^{\prime}{ }_{j}-c_{2}\left(h_{i}-h_{j}\right)^{2} / 2,
$$

where $h_{i}{ }^{\prime}=c_{0} / 2+c_{1} h_{i}+\left(c_{2} / 2\right) h_{i}^{2}$, as shown by Li et al. ${ }^{51}$ Eq. (6) explains the physical nature of this approximation: The hydrophobic potential $h^{\prime}$ is supplemented by the energy of demixing, well known from the Hildebrand theory of solutions that favors structures with spatial segregation of amino acids. Thus, we use the notation (Hp.Dx) for approximation (3b). In the next approximation [Eq. (3c)], the hydrophobicity is supplemented by electrostatic interactions, where electrostatics are linearly related to the experimental isoelectric points $\mathrm{pI}$ of amino acids, ${ }^{43-47}$ measured in $\mathrm{pH}$ units. This describes the abbreviation (Hp.pH) used for approximation (3c). The last approximation, (3d) (Hp.Dx.pH), contains all 3 elementshydrophobicity, energy of demixing, and electrostaticsand therefore actually gives the best results.

The numerical experiments performed for a variety of known CPs have shown that more complex approximations are not necessary, and the inclusion of higher order terms in the Taylor expansion of the function $e(h, q)$ did not in general lead to the significant increase of correlations with $E$.

\section{RESULTS AND DISCUSSION Pairwise Contact Potentials Studied}

In this article, we have studied mostly new potentials developed since 1995, and used by groups that were the most successful in predicting protein structures from the amino acid sequence in recent CASP experiments. We have included also a few older, historically important potentials. The total number of potentials analyzed in this work is 29 , listed and abbreviated as follows:

- TS-the oldest statistical potential derived by Tanaka and Scheraga. ${ }^{14}$ We also analyze the matrices N.TS = $\left(N_{i j}\right)$ and IN.TS $=\left[\log \left(N_{i j}\right)\right]$, where $N_{i j}$ is the number of contacts between amino acids $i$ and $j$.

- $\mathrm{RO}$ - the matrix developed by Robson and Osguthorpe. ${ }^{15}$ This potential has been applied by Bates and cowork$\mathrm{ers}^{52}$ for threading and used quite successfully in CASP5.

- BL-distance-dependent statistical potential proposed by Bryant and Lawrence. ${ }^{3}$ We have used the energies from the first bin only (contacts within $5 \AA$ ). Matrix BL has been used for threading, ${ }^{37}$ and most recently by Fang and Shortle ${ }^{35}$ in their ab initio method.

- TD-mixed quasi-optimization potential developed by Thomas and Dill. ${ }^{26}$

- MS-optimization-based potential derived by Mirny and Shakhnovich ${ }^{25}$ by the maximization of the harmonic mean of $Z$ scores for decoys.

- VD-effective optimization-based potential constructed on the perceptron criterion proposed by Vendruscolo and Domany ${ }^{28}$ The VD potential is based on the first optimization-derived potential of Maiorov and Crippen. ${ }^{24}$ The comparison of $\mathrm{MS}$ and $\mathrm{VD}$ is given in Vendruscolo et al. ${ }^{29}$

- BFKV-effective optimization-derived potential that is a modified version of VD. ${ }^{53}$

- MJ1, MJ1h, MJ2, MJ2h, MJ3, MJ3h-MiyazawaJernigan potentials published in $1985,{ }^{18} 1996,{ }^{21}$ and 1999. ${ }^{22}$ Each Miyazawa-Jernigan article contains a derivation of 2 potentials: one including energy of transfer of amino acids from water to the protein environment (those are marked with the suffix " $h$ "), and another for interactions in an average buried environment. Because MJ1h has a correlation of 0.97 with MJ2h, we have studied only MJ2h. A modified version of MJ1h potential has been used by Liwo and coworkers in the $a b$ initio UNRES method. ${ }^{17,38}$ Because the last potential has a correlation of 0.97 with $\mathrm{MJ} 1 \mathrm{~h}$, we have omitted it in the comparative analysis. Similarly, because potentials MJ2 and MJ3 are highly correlated (0.994), we have studied the newest potential, MJ3, only. It is worth mentioning that potentials MJ1h and $\mathrm{MJ} 2 \mathrm{~h}$ are the most frequently analyzed, modified, and used in protein structure predictions. $5,12,16,19,20,51,54,55$ Matrices N.MJ2 and IN.MJ2, with number of contacts and logarithms of the number of contacts, will also be investigated.

- BT-potential developed by Betancourt and Thirumalai, ${ }^{16}$ which is a modified version of MJ2h.

- TEl, TEs-effective optimization-derived potentials proposed by Tobi et al. ${ }^{27}$ based on the mixed perceptron- $Z$ score criterion. TEl and TEs are potentials obtained for large and small sets of decoys, respectively.

- MJPL, HLPL-potentials developed by Park and Levitt. ${ }^{5} \mathrm{MJPL}$ is a modified version of MJ1h, while HLPL is an improvement of an earlier potential of Hinds and 
TABLE I. Correlations between CPs for Lower Triangular Part and Distances Between Normalized Potentials for Upper Triangular Part, for a Convenience of Easy Comparison the Results are Multiplied by Factor 100 and the Coloring Scheme Explained Below the Table is Used. Potentials Derived by Optimization are Marked in Blue.

\begin{tabular}{|c|c|c|c|c|c|c|c|c|c|c|c|c|c|c|c|c|c|c|c|c|c|c|c|c|c|c|c|c|c|}
\hline Matrix & $\mathrm{Q}_{\mathrm{\theta}}$ & $\mathrm{Om}^{\mathrm{m}}$ & $G_{p}$ & HLPL & SKOb & $\mathrm{SKO}_{\mathrm{A}}$ & SIKG & M.PLL & M.sh & M/2h & TS & BT & BFKV & TD & TEI & TEs & $\mathrm{RO}$ & MS & MJ1 & MU3 & GKS & 82 & $B 1$ & $\mathrm{B3}$ & 85 & VD & BC & 84 & MS8M \\
\hline$a$ &. & 38 & 68 & 71 & 71 & 73 & 68 & 74 & 61 & 72 & 70 & 84 & 84 & 82 & 92 & 98 & 100 & 127 & 127 & 126 & 142 & 137 & 125 & 140 & 122 & 116 & 127 & 142 & 150 \\
\hline $\mathrm{am}$ & 98 & - & 44 & 50 & 66 & 66 & 67 & 81 & 69 & 87 & 83 & 77 & $\infty 0$ & 89 & 94 & 98 & 108 & 113 & 113 & 111 & 125 & 121 & 116 & 123 & 112 & 109 & 121 & 134 & 150 \\
\hline$\alpha_{p}$ & $\pi$ & 90 & . & 53 & 62 & 57 & 67 & 78 & 77 & 94 & 92 & 74 & 73 & 92 & 97 & 101 & 115 & 105 & 104 & 99 & 113 & 110 & 108 & 110 & 104 & 104 & 115 & 129 & 150 \\
\hline HLPL & 75 & 82 & 86 & . & 48 & 33 & 42 & 61 & 61 & $\infty$ & 73 & 61 & 68 & $\mathscr{2}$ & 92 & 98 & 114 & 101 & 101 & 95 & 114 & 112 & 100 & 114 & 100 & 98 & 111 & 127 & 150 \\
\hline skOb & 75 & 78 & 81 & 68 & . & 40 & 53 & 61 & 65 & 78 & 70 & 70 & 08 & 88 & 92 & 93 & 113 & 118 & 118 & 113 & 127 & 122 & 119 & 122 & 106 & 115 & 121 & 129 & 150 \\
\hline SKOa & 74 & 78 & 84 & 86 & $\mathscr{2}$ & - & 28 & 48 & 61 & 68 & 70 & 63 & 50 & 85 & 91 & 90 & 109 & 113 & 113 & 108 & 124 & 122 & 112 & 123 & 107 & 109 & 113 & 134 & 150 \\
\hline S.KG & 78 & 78 & 78 & 91 & 86 & 96 & . & 47 & 52 & $\infty$ & 64 & 61 & 65 & 83 & 89 & 98 & 105 & 116 & 115 & 110 & 129 & 126 & 115 & 126 & 111 & 108 & 114 & 134 & 150 \\
\hline U.JPL & 73 & 68 & 69 & 81 & 81 & 88 & 60 & - & 42 & 50 & 68 & 62 & 64 & 76 & 84 & 98 & 103 & 115 & 114 & 110 & 135 & 125 & 113 & 130 & 104 & 108 & 114 & 137 & 150 \\
\hline M.s.h & 81 & 76 & 70 & 81 & 79 & 81 & 38 & 91 & . & 47 & 64 & 53 & 65 & 72 & 80 & 90 & 97 & 111 & 112 & 105 & 132 & 122 & 114 & 126 & 102 & 107 & 113 & 133 & 150 \\
\hline u.seh & 74 & 62 & 56 & 68 & 70 & $\pi 7$ & 62 & 82 & 89 & . & 49 & 82 & $\pi$ & 81 & 93 & 104 & 109 & 137 & 138 & 135 & 157 & 148 & 138 & 148 & 127 & 120 & 128 & 146 & 150 \\
\hline TS & 78 & 68 & 58 & 73 & 75 & 76 & 80 & 77 & 80 & 88 & . & 91 & 83 & 88 & 98 & 106 & 115 & 139 & 139 & 140 & 156 & 151 & 141 & 148 & 130 & 123 & 132 & 145 & 150 \\
\hline BT & क5 & 70 & 73 & 81 & 75 & 80 & 82 & 81 & 86 & के & 59 & - & 63 & 86 & 89 & 93 & 105 & 87 & 85 & 74 & 105 & 85 & 89 & 102 & 85 & 96 & 98 & 122 & 150 \\
\hline GFKV & 65 & 68 & 73 & $\pi$ & 78 & 82 & 79 & 80 & 79 & 70 & 65 & 80 &. & 78 & 92 & 99 & 108 & 109 & 106 & 104 & 121 & 114 & 107 & 117 & 87 & 107 & 111 & 131 & 140 \\
\hline TD & कळ & 60 & 57 & 58 & 61 & 64 & के & 71 & 74 & का & 61 & 63 & 71 & $\cdot$ & 98 & 100 & 100 & 121 & 120 & 120 & 138 & 132 & 118 & 136 & 114 & 116 & 114 & 146 & 140 \\
\hline TEI & 58 & 58 & 53 & 58 & 57 & 59 & 60 & 64 & 68 & 57 & 52 & 61 & 57 & 52 & . & 92 & 112 & 119 & 118 & 114 & 129 & 122 & 119 & 125 & 113 & 118 & 120 & 136 & 140 \\
\hline TES & 52 & 52 & 48 & 52 & 56 & 51 & 52 & 52 & 60 & 46 & 45 & 57 & 51 & 41 & 57 & - & 118 & 118 & 116 & 113 & 128 & 121 & 119 & 120 & 108 & 115 & 126 & 127 & 140 \\
\hline RO & 50 & 42 & 34 & 38 & 38 & 41 & 45 & 47 & 53 & 41 & 34 & 44 & 42 & 49 & 37 & 31 & . & 120 & 121 & 118 & 131 & 128 & 114 & 133 & 103 & 124 & 125 & 137 & 150 \\
\hline MS & 19 & 36 & 45 & 49 & 30 & 36 & 33 & 34 & 38 & 7 & 4 & 63 & 41 & 27 & 30 & 31 & 28 & - & 24 & 56 & 66 & 68 & 71 & 80 & 82 & 82 & 98 & 113 & 150 \\
\hline us1 & 19 & 36 & 46 & 49 & 31 & 36 & 34 & 36 & 38 & 5 & 3 & 63 & 42 & 28 & 30 & 32 & 27 & 97 & . & 53 & 65 & 67 & 69 & 78 & 81 & 88 & 95 & 112 & 150 \\
\hline$M, 3$ & 21 & 39 & 51 & 55 & 36 & 42 & 40 & 40 & 45 & 9 & 2 & 73 & 46 & 29 & 36 & 36 & 31 & 85 & 65 & - & 50 & 45 & 55 & 66 & 72 & 93 & 91 & 108 & 150 \\
\hline GKS & -1 & 22 & 36 & 34 & 20 & 23 & 17 & 9 & 13 & .23 & 22 & 44 & 27 & 4 & 17 & 18 & 14 & 78 & 79 & 87 & . & 49 & 71 & 68 & 85 & 106 & 104 & 107 & 140 \\
\hline 82 & 6 & 27 & 39 & 37 & 25 & 26 & 22 & 22 & 26 & -10 & -14 & 55 & 35 & 13 & 26 & 27 & 17 & $\pi 7$ & $\pi$ & 89 & 88 & - & 78 & 57 & 80 & 109 & 106 & 100 & 140 \\
\hline 81 & 21 & 33 & 42 & 50 & 29 & 37 & 34 & 36 & 35 & 5 & 0 & 60 & 43 & 31 & 29 & 30 & 34 & 75 & 78 & 86 & 75 & 70 & . & 91 & 75 & 58 & 86 & 119 & 140 \\
\hline 83 & 3 & 25 & 40 & 35 & 26 & 24 & 20 & 16 & 21 & $\rightarrow$ & -10 & 48 & 32 & 7 & 22 & 28 & 12 & 68 & 70 & 78 & 78 & 83 & 50 & - & $\pi 7$ & 115 & 114 & 91 & 150 \\
\hline 205 & 28 & 37 & 46 & 50 & 43 & 43 & 38 & 46 & 48 & 20 & 18 & 63 & 62 & 36 & 36 & 41 & 47 & 68 & 87 & 74 & 63 & 68 & 72 & 70 & . & 109 & 111 & 102 & 140 \\
\hline VD & 33 & 41 & 46 & 51 & 34 & 41 & 41 & 42 & 43 & 28 & 24 & 54 & 43 & 33 & 31 & 34 & 24 & 酷 & 61 & 56 & 45 & 41 & 52 & 34 & 41 & $\cdot$ & 110 & 131 & 160 \\
\hline BL & 19 & 26 & 33 & 38 & 27 & 36 & 35 & 35 & 36 & 18 & 12 & 52 & 38 & 38 & 28 & 21 & 22 & 52 & 55 & 58 & 45 & 44 & 63 & 35 & 39 & 39 & . & 137 & 150 \\
\hline 84 & -1 & 10 & 17 & 20 & 17 & 11 & 10 & 6 & 12 & -7 & -5 & 25 & 14 & -7 & 9 & 20 & 6 & 36 & 37 & 41 & 43 & 48 & 29 & 58 & 48 & 15 & 6 & - & 150 \\
\hline usam & .12 & -12 & -10 & .18 & .18 & .15 & -16 & -16 & .14 & -11 & -12 & .17 & -1 & 2 & 4 & 4 & .6 & -15 & .15 & 9 & -2 & 0 & .2 & .5 & .3 & .24 & .14 & 6 & . \\
\hline
\end{tabular}

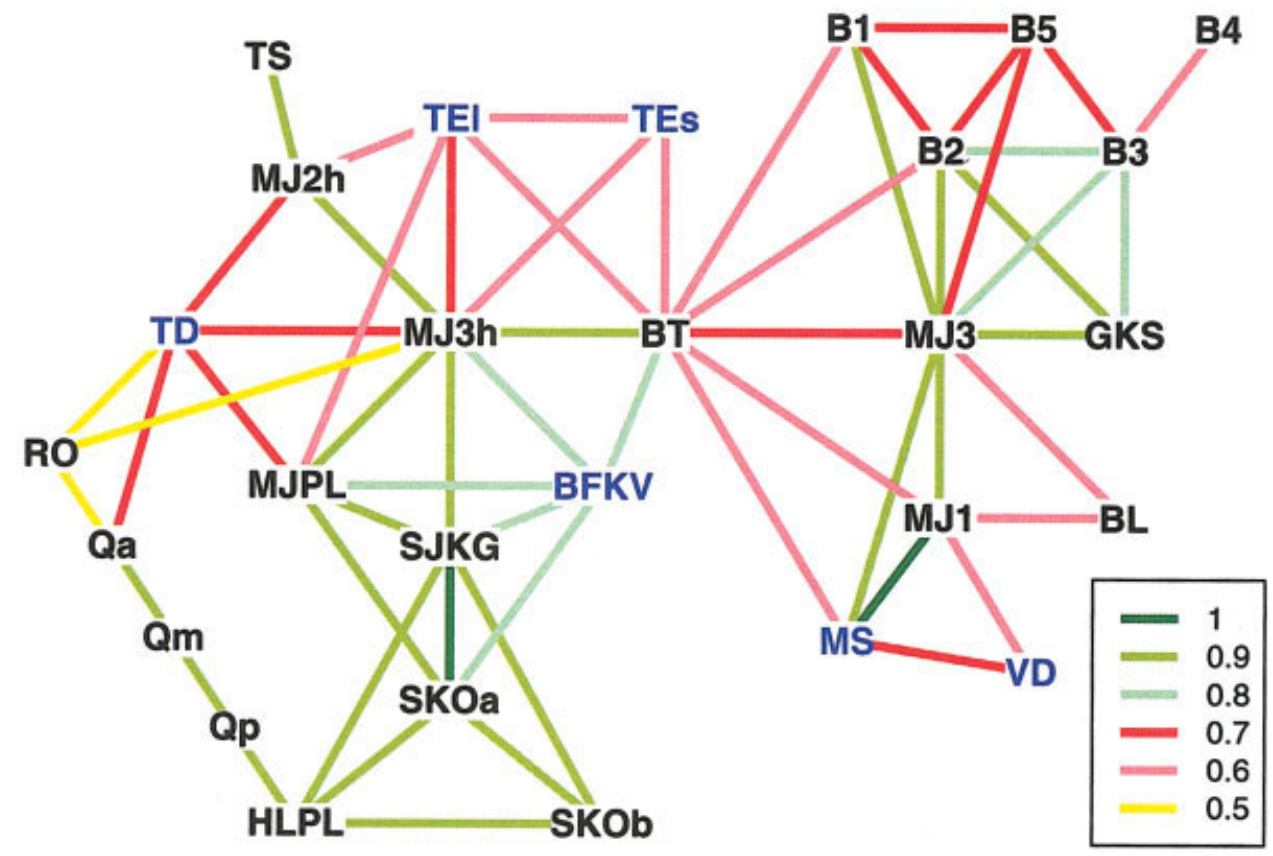

Fig. 1. Graphical illustration of correlations among different protein potentials. Coloring scheme is the same as in Table I.

Levitt. ${ }^{2}$ These CPs are part of a hierarchical method of ab initio protein structure prediction. ${ }^{41}$

- GKS-quasi-chemical statistical potential of Godzik et al. ${ }^{4}$
- SJKG, SKOa, SKOb-quasi-chemical CPs of Skolnick et al. ${ }^{8,11}$

- Qa, Qm, Qp-new quasi-chemical potentials developed by Kolinski and coworkers, ${ }^{13}$ which depend on the 
relative orientation of side-chains of 2 contacting residues. Three different possible mutual orientations of the interacting side groups (a, antiparallel; $\mathrm{m}$, intermediate; p, parallel) were considered. ${ }^{13} \mathrm{Qa}, \mathrm{Qm}$, and Qp potentials were used in TOUCHSTONE, one of the most effective methods for structure prediction, as proven during the CASP5 experiment. ${ }^{40}$ Additionally, such environment-dependent potentials may include both the group orientation and the two-state (compact/ extended) main-chain conformation information. ${ }^{23}$ Since it leads to $40 \times 40$ matrices $\mathrm{QaS}, \mathrm{QmS}$, and $\mathrm{QpS}$, these generalized potentials are not included here in the comparative analysis.

- B1,..,B5-the newest version of quasi-chemical potential developed in the research group of Baker. Earlier versions of this potential were discussed in Simons et al. ${ }^{9,10}$ The potential is distance dependent: Distance bins are denoted by increasing integer numbers. The potentials are a part of ROSETTA, currently the most successful protocol for ab initio prediction of protein structure from sequence. ${ }^{34,39}$

- MSBM-optimization-derived potential developed by Micheletti et al. ${ }^{30}$

\section{Comparative Analysis of One-Body Approximation for Pairwise Contact Potentials}

Table I shows the results of calculations performed for all of the 29 potentials and matrices with numbers of contacts. The entries below the diagonal show correlation coefficients (cor) between potentials, while the entries above the diagonal list the mean Euclidean distances between the normalized potentials (dist). Potentials developed by the optimization methods are marked in blue. Figure 1 graphically illustrates the results from Table I. Each of the 28 potentials listed is represented by a node of the graph. (MSBM, N.TS, IN.TS, N.MJ2 and IN.MJ2 are not included because of their small correlations with other potentials.) All the strongest correlations of the order 0.9-1.0 are visualized as graph edges. Lower correlations have not been shown for reasons of clarity, since, often, if $\operatorname{cor}(a, b) \geq 0.9$ and $\operatorname{cor}(b, c) \geq 0.9$, then $\operatorname{cor}(a, c) \geq 0.8$. In the case where a given node (potential) is not correlated with other nodes by at least a value of 0.9 , we show the first 2-4 edges connecting to nodes with the highest correlation (we use colors to indicate the different ranges of correlation). Table I and Figure 1 show that CPs can be clustered into 2 groups. The first cluster is centered on MJ3h and SJKG, and the second one around MJ3. Using a rule, that each potential in the group has to be correlated at the level of at least 0.9 with a neighbor, the following 2 sets clearly arise: \{TS, MJ2h, MJ3h, MJPL, SJKG, SKOa, SKOb, HLPL, Qp, Qa, Qm, BT\}, \{MJ3, MJ1, MS, GKS, B1, B2\}. Potentials in the second set (except MS) were designed to diminish the influence of hydrophobic interactions, by considering contacts for buried residues only, and by a proper definition of the reference state.

Table II shows cor, dist, and err between analyzed potentials and their one-body approximations. By comparing columns $(\mathrm{Hp})$ with $(\mathrm{Hp} . \mathrm{Dx})$ and $(\mathrm{Hp} . \mathrm{pH})$ with $(\mathrm{Hp}-$
.Dx.pH), we can estimate demixing energies, while the comparison of columns $(\mathrm{Hp})$ with $(\mathrm{Hp} . \mathrm{pH})$ and $(\mathrm{pH} . \mathrm{Hp})$, and (Hp.Dx) with (Hp.Dx.pH) enables the evaluation of the strength of electrostatics in the protein potentials. Columns 17-19 contain errors of approximation by the formula (Hp.Dx.pH) for suboptimal solutions $(h, q)$ that have significant correlations with hydrophobicity $(\mathrm{Hp})$ and isoelectric points ( $\mathrm{pH})$. Column 20 of Table II shows correlations between approximating vectors $h$ from the formula (Hp.Dx.pH) and the closest hydrophobicity scale (with negative sign). Forty hydrophobicity scales with correlations greater than 0.68 compared to the Kyte-Doolittle scale were selected from literature. Column 21 contains identifying numbers of the closest hydrophobicity scales. All numerical data and detailed references are available as Supplementary Materials to this article, which can be found at http://www.mimuw.edu.pl/ pokar. The last column in Table II displays correlations between vectors $q$ and isoelectric points of amino acids pI $(\mathrm{pH}){ }^{43-47}$ The major conclusions from the analysis of Table II are as follows:

- All potentials (except TEl, TEs, VD, B4, and MSBM) can be quite well approximated by simple functions of one-body factors $h$ and $q$ that are highly correlated with hydrophobicities and isoelectric points of amino acids. Indeed, a correlation between approximating vectors $h$ and the closest hydrophobic scale is roughly 0.9 , which is more than a mean correlation between 2 different hydrophobic scales. Because of this we may interpret vectors $h$ as statistical hydrophobicity scales. Hydrophobicity is the most dominant factor in protein potentials, much more important than electrostatics or demixing energy.

- By comparing rows in Table II, we may group together potentials having similar characteristics, similar to what was done earlier with Table I. The first group of potentials (rows $\mathrm{Qa}-\mathrm{BFKV}$ ) is dominated by the onebody transfer energy. Indeed, the correlation coefficients with the simplest approximation ( $\mathrm{Hp})$ are mostly above 0.9 , values only slightly smaller than the correlations with (Hp.Dx.pH). The contributions from demixing and electrostatics are negligible. The nearest hydrophobicity scale to the vectors $h$ from this group is the Wertz-Scheraga frequency of occurrence for a given type residue inside proteins (scale no. 26). The second group of potentials (rows MS-B5) is poorly approximated by the $(\mathrm{Hp})$ formula (correlation coefficient range is from 0.2 to 0.3 , except 0.5 for GKS). Using the (Hp.Dx) formula, the correlation increases to about 0.8 , and to about 0.9 for the (Hp.Dx.pH) formula. In the last formula the $c_{1}$ coefficients are negative, while the $c_{2}$ coefficients have positive values. Thus, the (Hp.Dx.pH) formula can be written in simplified form as

$$
\tilde{e}(h, q)_{i, j}=c_{0}-h_{i} h_{j}+q_{i} q_{j} .
$$

The large contributions of the demixing term means that the $c_{0}-h_{i} h_{j}$ term describes the effects of interactions already present within the protein environment, 
TABLE II. One-Body Approximations to Protein Contact Potentials (CPs)

\begin{tabular}{|c|c|c|c|c|c|c|c|c|c|c|c|c|c|c|c|c|c|c|c|c|c|}
\hline \multirow[t]{2}{*}{ Matrix } & \multicolumn{3}{|c|}{$\mathrm{Hp}$} & \multicolumn{3}{|c|}{ Hp.Dx } & \multicolumn{3}{|c|}{ Hp.pH } & \multicolumn{3}{|c|}{ pH.Hp } & \multicolumn{3}{|c|}{ Hp.Dx.pH } & \multicolumn{3}{|c|}{ Hp.Dx.pH ${ }^{*}$} & \multirow[t]{2}{*}{ cor.Hp } & \multirow[t]{2}{*}{ nb. Hp } & \multirow[t]{2}{*}{ cor.pH } \\
\hline & cor & dist & err & cor & dist & err & cor & dist & err & cor & dist & err & $\infty$ & dist & err & cor & dist & err & & & \\
\hline$\overline{\overline{Q a}}$ & $\overline{91}$ & 42 & 44 & 91 & 42 & 41 & 95 & 32 & 31 & 95 & 32 & 31 & 95 & 31 & 30 & 95 & 31 & 30 & $\overline{76}$ & $\overline{27}$ & $\overline{62}$ \\
\hline $\mathrm{am}_{\mathrm{n}}$ & 86 & 53 & 51 & 86 & 53 & 51 & 91 & 43 & 42 & 91 & 43 & 42 & 93 & 38 & 37 & 91 & 43 & 42 & 66 & 26 & 60 \\
\hline HLPL & 82 & 59 & 42 & 83 & 58 & 41 & 85 & 54 & 38 & 93 & 38 & 28 & 93 & 38 & 28 & 86 & 53 & 38 & 74 & 26 & 91 \\
\hline SKOb & 91 & 43 & 40 & 91 & 42 & 39 & 96 & 41 & 38 & 96 & 28 & 27 & 97 & 24 & 13 & 92 & 41 & 37 & 84 & 26 & 89 \\
\hline SKOa & 86 & 53 & 51 & 87 & 51 & 49 & 89 & 48 & 46 & 94 & 33 & 33 & 95 & 32 & 33 & 90 & 45 & 44 & 85 & 26 & 86 \\
\hline SJKG & 88 & 50 & 48 & 89 & 47 & 45 & 90 & 46 & 44 & 95 & 32 & 31 & 95 & 32 & 31 & 91 & 41 & 40 & 92 & 36 & 88 \\
\hline MJPL & 88 & 50 & 11 & 90 & 44 & 10 & 90 & 46 & 10 & 95 & 32 & 7 & 95 & 32 & 7 & 93 & 38 & 8 & 93 & 12 & 84 \\
\hline MJ3h & 86 & 53 & 49 & 93 & 39 & 37 & 88 & 49 & 46 & 96 & 30 & 28 & 96 & 30 & 28 & 95 & 31 & 30 & 97 & 19 & 92 \\
\hline MJZh & 98 & 19 & 8 & 99 & 14 & 6 & 99 & 14 & 6 & 99 & 11 & 5 & 99 & 11 & 5 & 99 & 11 & 5 & 97 & 25 & 92 \\
\hline TS & 98 & 18 & 4 & 98 & 17 & 4 & 99 & 16 & 4 & 99 & 17 & 4 & 99 & 16 & 4 & 99 & 16 & 4 & 87 & 25 & 91 \\
\hline BT & 62 & 87 & 77 & 82 & 59 & 56 & 69 & 78 & 71 & 88 & 48 & 46 & 90 & 46 & 44 & 90 & 46 & 44 & 95 & 25 & 93 \\
\hline BFKV & 71 & 76 & 69 & 80 & 63 & 58 & 74 & 72 & 66 & 88 & 49 & 46 & 88 & 49 & 46 & 85 & 55 & 52 & 92 & 12 & 79 \\
\hline TD & 72 & 75 & 66 & 75 & 71 & 63 & 77 & 68 & 60 & 79 & 66 & 59 & 82 & 60 & 54 & 80 & 63 & 57 & 93 & 34 & 82 \\
\hline TEl & 59 & 91 & 80 & 61 & 89 & 79 & 66 & 82 & 75 & 71 & 76 & 70 & 71 & 76 & 70 & 69 & 79 & 72 & 93 & 19 & 76 \\
\hline TEs & 56 & 94 & 82 & 64 & 84 & 76 & 61 & 89 & 79 & 71 & 76 & 70 & 71 & 76 & 70 & 69 & 79 & 71 & 83 & 26 & 67 \\
\hline MS & 22 & 125 & 97 & 68 & 80 & 73 & 42 & 108 & 91 & 71 & 76 & 70 & 79 & 65 & 61 & 79 & 65 & 62 & 95 & 1 & 94 \\
\hline MJ1 & 22 & 125 & 13 & 70 & 78 & 9 & 41 & 109 & 12 & 72 & 74 & 9 & 80 & 63 & 8 & 80 & 63 & 8 & 95 & 1 & 94 \\
\hline MJ3 & 21 & 126 & 98 & 80 & 63 & 60 & 41 & 109 & 91 & 82 & 60 & 57 & 90 & 45 & 44 & 90 & 45 & 44 & 94 & 1 & 95 \\
\hline GKS & 50 & 100 & 86 & 85 & 56 & 53 & 56 & 93 & 82 & 87 & 50 & 48 & 90 & 44 & 43 & 90 & 44 & 43 & 96 & 1 & 95 \\
\hline$B 2$ & 34 & 115 & 94 & 86 & 52 & 51 & 46 & 104 & 89 & 88 & 48 & 47 & 94 & 35 & 35 & 94 & 35 & 35 & 96 & 1 & 95 \\
\hline B1 & 21 & 126 & 98 & 68 & 80 & 73 & 50 & 78 & 72 & 83 & 58 & 55 & 83 & 58 & 55 & 83 & 58 & 55 & 93 & 34 & 91 \\
\hline B3 & 31 & 117 & 95 & 86 & 52 & 50 & 35 & 114 & 93 & 88 & 50 & 48 & 89 & 46 & 45 & 89 & 46 & 45 & 97 & 1 & 93 \\
\hline 85 & 26 & 122 & 96 & 87 & 51 & 50 & 26 & 122 & 96 & 88 & 50 & 48 & 98 & 17 & 4 & 87 & 51 & 49 & 98 & 5 & 98 \\
\hline VD & 29 & 119 & 96 & 47 & 103 & 88 & 45 & 105 & 90 & 55 & 94 & 83 & 59 & 90 & 80 & 59 & 90 & 80 & 77 & 36 & 86 \\
\hline BL & 10 & 134 & 100 & 52 & 97 & 85 & 51 & 99 & 86 & 53 & 97 & 85 & 75 & 71 & 66 & 75 & 71 & 66 & 93 & 36 & 71 \\
\hline B4 & 28 & 120 & 96 & 64 & 85 & 76 & 68 & 80 & 73 & 64 & 85 & 76 & 86 & 53 & 38 & 64 & 85 & 76 & 85 & 30 & 100 \\
\hline MSBM & 33 & 116 & 94 & 66 & 82 & 75 & 66 & 82 & 75 & 66 & 82 & 75 & 100 & 8 & 2 & 66 & 82 & 75 & 51 & 9 & 100 \\
\hline N.TS & 84 & 57 & 36 & 91 & 43 & 28 & 84 & 57 & 36 & 91 & 44 & 28 & 91 & 43 & 27 & 91 & 43 & 27 & 66 & 1 & 100 \\
\hline IN.TS & 89 & 47 & 10 & 89 & 47 & 10 & 89 & 47 & 10 & 88 & 49 & 10 & 90 & 46 & 10 & 82 & 59 & 12 & 67 & 1 & 100 \\
\hline N.MJ2 & 82 & 60 & 37 & 91 & 43 & 27 & 82 & 60 & 37 & 90 & 44 & 28 & 92 & 41 & 26 & 92 & 41 & 26 & 66 & 1 & 100 \\
\hline IN.MJ2 & 91 & 43 & 4 & 91 & 42 & 4 & 91 & 42 & 4 & 91 & 43 & 4 & 93 & 34 & 3 & 86 & 52 & 4 & 66 & 1 & 100 \\
\hline
\end{tabular}

50

60

80

90

100

Columns 2-16 contain values of optimal cor, dist, and err between particular CPs and their approximations. Columns 17-19 contain values of cor, dist, and err for suboptimal solutions $(h, q)$, which have significant correlations with hydrophobicity (column 20 ) and isoelectric points pI (column 22). Column 21 contains numbers designating the closest hydrophobicity scales (details explained in the text). The scaling factor and the coloring scheme are the same as in Table I.

where similar residues (hydrophobic or polar) are pairwise attractive, while the interactions between polar and hydrophobic residues are repulsive. Interestingly, similar interactions are necessary for proteinlike folding thermodynamics in our minimal model of proteins. ${ }^{48,49}$ The most correlated hydrophobicity scale with solutions for this group of potentials is the popular Kyte-Doolittle scale (scale no. 1).

- Potentials developed by optimization methods have significantly less hydrophobic character than do the quasichemical potentials, and additionally are less stabilizing. The only exceptions are MS (with correlation 0.97 with MJ1) and BFKV (with correlation of order 0.8 with MJ3h, MJPL and SJKG). Optimization-based approaches may additionally lead to many surprising counterintuitive results. For example, in the MSBM potential, the energy of the contact TRP-MET exceeds more than 20 times all other contact energies. That may be a reason that such potentials are seldom used with much success for fold recognition, prediction of protein structure, or docking.
- Let us take a closer look at the potentials MJ3h, MJ3 and SJKG, which are centers of the groups. Potential MJ3h was derived by using the formula $e_{i j}=-\log \left(N_{i j} /\right.$ $\left.C_{i j}\right)-h_{i}-h_{j}$, where $N_{i j}$ denotes the number of observed contacts and $C_{i j}$ the number of expected contacts between residues $i$ and $j$, while $h_{i}$ is a one-body potential highly correlated with hydrophobicity (" $h$ " in the name of the potential refers to its hydrophobicity-driven nature). On the other hand, potential MJ3 has been derived from the formula $e_{i j}=-\log \left(N_{i j} / C_{i j}\right)$, that could allow us to estimate the influence of $h$ terms on the one-body approximation. Interestingly, the potential MJ3 have a relatively negligible correlation with the Wertz-Scheraga scale, however, the results of our approximation show that hydrophobicity still remains, in a form highly correlated with the Kyte-Doolittle scale.

- By comparing potentials SJKG, SKOa, and SKOb, one may notice that the composition corrected potential (SKOb) that was derived to increase its specificity is actually less specific than the simplest quasi-chemical potential obtained from the same set of protein struc- 
tures (SJKG), though, of course, it could be more effective for the prediction of protein structure. We define here the specificity of CPs, through low correlations with their one-body approximations. We may notice that potentials SKOb and Qa are roughly equivalent, which means that an antiparallel orientation does not add to the specificity. The specificity of two-body interaction is, however, increased for parallel orientations (compare SKOb with Qp). Also, the inclusion of the backbone geometry characteristics increases specificity of the potential (data not shown).

The last four rows of Table II show the correlation between the number of contacts $N$ or as $\log N$ and one-body approximations for potentials TS and MJ2. It is seen that the frequency of contacts of amino acids can be well approximated by hydrophobicity and electrostatic properties. Note that hydrophobicity and electrostatics in the CPs result not from sophisticated manipulations of the reference state (extensively studied in the past in the literature) but simply from the frequencies of contacts in protein structures. Interestingly, vectors $h$ approximating $N$ or $\log N$ correlate less with hydrophobicity than vectors $h$ approximating CPs. To the contrary, vectors $q$ approximating $N$ or $\log N$ correlate more strongly with $\mathrm{pI}(\mathrm{pH})$ than do the corresponding vectors approximating CPs. Comparison of columns (Hp.Dx) and (Hp.Dx.pH) shows that electrostatic interactions are almost negligible.

Approximation of the CPs by one-body amino acid functions was studied earlier by Godzik et al. ${ }^{4}$ and by Li et al. ${ }^{51}$ The present results are, however, stronger and more universal. The main point of the work of Godzik et al. ${ }^{4}$ was to compare known potentials and to discover their relationships with hydrophobicity (Table II). Their second aim, namely, the derivation of the excess part of the potential $e_{i j}^{\text {excess }}=e_{i j}-e_{i j}^{\text {ideal }}$, where $e_{i, j}^{\text {ideal }}=\left(e_{i i}+e_{j j}\right) / 2$ was not completed, as can be seen clearly from our present results. The authors found that the correlation of GKS with $e_{i, j}^{\text {ideal }}$ is only 0.21 , and that led to their mistaken conclusion that their potential was more specific than, for example, the TS potential having a correlation 0.98 . The reason for this poor correlation lies in the formulation of the ideal values. Indeed, our Table II shows that GKS potential can be well approximated by one-body functions by including both hydrophobicity and electrostatics.

The major advantage of the work of $\mathrm{Li}$ et al. ${ }^{51}$ was the derivation of a better (in general) approximating vector than $\left(e_{i i}\right)$ and a richer approximating formula (Hp.Dx). The approximating vector they used was the eigenvector of the dominant eigenvalue of the matrix obtained from the matrix of the potential with the mean value subtracted. However, such an approximation can sometimes be significantly worse than the optimal one (e.g., for MSBM, we obtain in this way cor $=0.66$ instead of 0.997 , which was found with the optimization formula Hp.Dx.pH).

\section{CONCLUSIONS}

It has been shown that all analyzed CPs can be divided into two groups, regardless of having completely different derivation origin. Most of these knowledge-based statisti- cal potentials could be well approximated by appropriate combinations of one-body components. The one body approximation suggests the two following ideal amino acid interaction forms:

- Let $h$ be a vector composed of the normalized WertzScheraga interior frequency coefficients with negative signs. Then the formula $e_{i j}=h_{i}+h_{j}$ gives a potential that belongs to the first group (e.g., correlation with MJ2h and TS are 0.90 and 0.88 , respectively).

- Let $h$ be a vector composed of the normalized KyteDoolittle coefficients with negative signs and $q$ be the normalized isoelectric point $(\mathrm{pH})$ vector. Then the potential $e_{i j}=-h_{i} h_{j}+0.5 q_{i} q_{j}$ correlates moderately well with members of the second group of CPs (cor $=0.66$, 0.60 , and 0.59 for $\mathrm{MJ} 3, \mathrm{MJ} 1$, and $\mathrm{B} 2$, respectively).

From a practical point of view, the accurate one-body approximations of CPs provided in this work could be very useful is some applications, especially for 3-dimensional threading algorithms. On the other hand the lack of "excess" contributions to the pairwise potentials (that cannot be approximated by the one-body component) strongly suggests that an efficient structure-specific, knowledge-based pairwise potential is still to be designed. This means that there are opportunities to develop different further types of potentials (perhaps multibody).

\section{ACKNOWLEDGMENTS}

Our thanks to Sanzo Miyazawa for helpful discussions, and to the Reviewers for insightful comments.

\section{REFERENCES}

1. Sippl MJ. Calculation of conformational ensembles from potentials of mean force- an approach to the knowledge-based prediction of local structures in globular proteins. J Mol Biol 1990;213:859-883.

2. Hinds DA, Levitt M. Exploring conformational space with a simple lattice model for protein structure. J Mol Biol 1994;243:668-682.

3. Bryant SH, Lawrence CE. An empirical energy function for threading protein-sequence through the folding motif. Proteins 1993;16:92-112.

4. Godzik A, Kolinski A, Skolnick J. Are proteins ideal mixtures of amino acids?: analysis of energy parameter sets. Protein Sci 1995;4:2107-2117.

5. Park B, Levitt M. Energy functions that discriminate X-ray and near-native folds from well-constructed decoys. J Mol Biol 1996;258: 367-392.

6. Bahar I, Jernigan RL. Inter-residue potentials in globular proteins and the dominance of highly specific hydrophilic interactions at close separation. J Mol Biol 1997;266:195-214.

7. Liwo A, Pincus MR, Wawak RJ, Rackovsky S, Oldziej S, Scheraga HA. A united-residue force field for off-lattice protein-structure simulations: 2. Parameterization of short-range interactions and determination of weights of energy terms by $Z$-score optimization. J Computational Chem 1997;18:874-887.

8. Skolnick J, Jaroszewski L, Kolinski A, Godzik A. Derivation and testing of pair potentials for protein folding: When is the quasichemical approximation correct? Protein Sci 1997;6:676-688.

9. Simons KT, Kooperberg C, Huang E, Baker D. Assembly of protein tertiary structures from fragments with similar local sequences using simulated annealing and Bayesian scoring functions. J Mol Biol 1997;268:209-225.

10. Simons KT, Ruczinski I, Kooperberg C, Fox BA, Bystroff C, Baker D. Improved recognition of native-like protein structures using a combination of sequence-dependent and sequence-independent features of proteins. Proteins 1999;34:82-95.

11. Skolnick J, Kolinski A, Ortiz A. Derivation of protein-specific pair 
potentials based on weak sequence fragment similarity. Proteins 2000;38:3-16

12. Zhang C, Kim S-H. Environment-dependent residue contact energies for proteins. Proc Natl Acad Sci USA 2000;97:2550-2555.

13. Boniecki M, Rotkiewicz P, Skolnick J, Kolinski A. Protein fragment reconstruction using various modeling techniques. J Comput Aided Mol Des 2003;17:725-738.

14. Tanaka S, Scheraga HA. Medium- and long-range interaction parameters between amino acids for predicting three-dimensional structures of proteins. Macromolecules 1976;9:945-950.

15. Robson B, Osguthorpe DJ. Refined models for computer-simulation of protein folding-applications to the study of conserved secondary structure and flexible hinge points during the folding of pancreatic trypsin-inhibitor. J Mol Biol 1979;132:19-51.

16. Betancourt MR, Thirumalai D. Pair potentials for protein folding: choice of reference states and sensitivity of predicted native states to variations in the interaction schemes. Protein Sci 1999;8:361-369.

17. Liwo A, Oldziej S, Pincus MR, Wawak RJ, Rackovsky S, Scheraga HA. A united-residue force field for off-lattice protein-structure simulations: 1. Functional forms and parameters of long-range side-chain interaction potentials from protein crystal data. J Comput Chem 1997;18:849-873.

18. Miyazawa S, Jernigan RL. Estimation of effective interresidue contact energies from protein crystal structures-quasi-chemical approximation. Macromolecules 1985;18:534-552.

19. Park BH, Huang ES, Levitt M. Factors affecting the ability of energy functions to discriminate correct from incorrect folds. J Mol Biol 1997;266:831-846.

20. Keskin O, Bahar I, Badretdinov OB, Ptitsyn OB, Jernigan RL. Empirical solvent-mediated potentials hold for both intramolecular and inter-molecular inter-residue interactions. Protein Sci 1998;7:2578-2586.

21. Miyazawa S, Jernigan RL. Residue-residue potentials with a favorable contact pair term and an unfavorable high packing density term, for simulation and threading. J Mol Biol 1996;256:623-644.

22. Miyazawa S, Jernigan RL. Self-consistent estimation of interresidue protein contact energies based on an equilibrium mixture approximation of residues. Proteins 1999;34:49-68.

23. Kolinski A. Protein modeling and structure prediction with a reduced representation. Acta Biochim Pol 2004;51:349-371.

24. Maiorov VN, Crippen GM. contact potential that recognizes the correct folding of globular proteins. J Mol Biol 1992;227:876-888.

25. Mirny LA, Shakhnovich EI. How to derive a protein folding potential?: a new approach to an old problem. J Mol Biol 1996;264: $1164-1179$.

26. Thomas PD, Dill KA. An iterative method for extracting energylike quantities from protein structures. Proc Natl Acad Sci USA 1996;93:11628-11633.

27. Tobi D, Shafran G, Linial N, Elber R. On the design and analysis of protein folding potentials. Proteins 2000;40:71-85.

28. Vendruscolo M, Domany E. Pairwise contact potentials are unsuitable for protein folding. J Chem Phys 1998;109:11101-11108.

29. Vendruscolo M, Mirny LA, Shakhnovich EI, Domany E. Comparison of two optimization methods to derive energy parameters for protein folding: Perceptron and $Z$ score. Proteins 2000;41:192201.

30. Micheletti C, Seno F, Banavar JR, Maritan A. Learning effective amino acid interactions through iterative stochastic techniques. Proteins 2001;42:422-431.

31. Sippl MJ. Knowledge-based potentials for proteins. Curr Opin Struct Biol 1995;5:229-235.

32. Hao MH, Scheraga HA. Designing potential energy functions for protein folding. Curr Opin Struct Biol 1999;9:184-188.

33. Buchete NV, Straub JE, Thirumalai D. Orientational potentials extracted from protein structures improve native fold recognition. Protein Sci 2004;13:862-874.

34. Bradley P, Chivian D, Meiler J, Misura KM, Rohl C, Schief W, Wedemeyer WJ, Schueler-Furman O, Murphy P, Schonbrun J, Strauss C, Baker D. Rosetta predictions in CASP5: Successes, failures, and prospects for complete automation. Proteins 2003;53: 457-468.

35. Fang QJ, Shortle D. Prediction of protein structure by emphasizing local side-chain/backbone interactions in ensembles of turn fragments. Proteins 2003;53:486-490.

36. Jones DT, McGuffin LJ. Assembling novel protein folds from supersecondary structural fragments. Proteins 2003;53:480-485.

37. Panchenko AR, Marchler-Bauer A, Bryant SH. Combination of threading potentials and sequence profiles improves fold recognition. J Mol Biol 2000;296:1319-1331.

38. Pillardy J, Czaplewski C, Liwo A, Lee J, Ripoll DR, Kamierkiewicz R, Odziej S, Wedemeyer WJ, Gibson KD, Arnautova YA, Saunders J, Ye YJ, Scheraga HA. Recent improvements in prediction of protein structure by global optimization of a potential energy function. Proc Natl Acad Sci USA2001;98:2329-2333.

39. Shao Y, Bystroff C. Predicting interresidue contacts using templates and pathways. Proteins 2003;53:497-502.

40. Skolnick J, Zhang Y, Arakaki AK, Kolinski A, Boniecki A, Szilagyi A, Kihara D. TOUCHSTONE: a unified approach to protein structure prediction. Proteins 2003;53:469-479.

41. Xia Y, Huang ES, Levitt M, Samudrala R. Ab initio construction of protein tertiary structures using a hierarchical approach. J Mol Biol 2000;300:171-185.

42. Munson PJ, Singh RK. Statistical significance of hierarchical multi-body potentials based on Delaunay tessellation and their application in sequence-structure alignment. Protein Sci 1997;6: $1467-1481$.

43. Georgescu RE, Alexov EG, Gunner MR. Combining conformational flexibility and continuum electrostatics for calculating pK(a)s in proteins. Biophys J 2002;83:1731-1748.

44. Mehler EL, Fuxreiter M, Simon I, Garcia-Moreno EB. The role of hydrophobic microenvironments in modulating pKa shifts in proteins. Proteins 2002;48:283-292.

45. Sandberg L, Edholm O. A fast and simple method to calculate protonation states in proteins. Proteins 1999;36:474-483.

46. Tollinger M, Crowhurst KA, Kay LE, Forman-Kay JD. Sitespecific contributions to the $\mathrm{pH}$ dependence of protein stability. Proc Natl Acad Sci USA 2003;100:4545-4550.

47. Laurents DV, Huyghes-Despointes BMP, Bruix M, Thurlkill RL, Schell D, Newsom S, Grimsley GR, Shaw KL, Trevi S, Rico M, Briggs JM, Antosiewicz JM, Scholtz JM, Pace CN. Charge-charge interactions are key determinants of the $\mathrm{pK}$ values of ionizable groups in ribonuclease $\mathrm{Sa}(\mathrm{pI}=3.5)$ and a basic variant $(\mathrm{pI}=$ 10.2). J Mol Biol 2003;325:1077-1092

48. Pokarowski P, Kolinski A, Skolnick J. A minimal physically realistic protein-like lattice model: designing an energy landscape that ensures all-or-none folding to a unique native state. Biophys J 2003;84:1518-1526.

49. Pokarowski P, Droste K, Kolinski A. A minimal protein-like lattice model: an alpha-helix motif. 2004. Submitted for publication.

50. Rao CR. Linear statistical inference and its applications. New York: Wiley; 1973.

51. Li H, Tang C, Wingreen NS. Nature of driving force for protein folding: a result from analyzing the statistical potential. Phys Rev Lett 1997;79:765-768.

52. Contreras-Moreira B, Fitzjohn PW, Offman M, Smith GR, Bates PA. Novel use of a genetic algorithm for protein structure prediction: searching template and sequence alignment space. Proteins 2003;53:424-429.

53. Bastolla U, Farwer J, Knapp EW, Vendruscolo M. How to guarantee optimal stability for most representative structures in the Protein Data Bank. Proteins 2001;44:79-96.

54. Du R, Grosberg AY, Tanaka T. Models of protein interactions: how to choose one. Fold Des 1998;3:203-211.

55. Gan HH, Tropsha A, Schlick T. Lattice protein folding with two and four-body statistical potentials. Proteins 2001;43:161-174.

\section{APPENDIX}

1. In order to prove the relation $(\mathrm{Hp}) \rightarrow(\mathrm{Hp} . \mathrm{Dx})$, let us first notice that (Hp.Dx) is equivalent to the following formula:

$$
a_{0}+a_{1}\left(h_{i}{ }^{\prime}+h_{j}{ }^{\prime}\right)+a_{2} h_{i}{ }^{\prime} h_{j}{ }^{\prime} \cdot(\text { Hp.Dx.2) }
$$

Obviously (Hp.Dx.2) can be transformed to (Hp.Dx) with $a_{0}:=c_{0}, a_{1}:=0, a_{2}:=c_{1}, h^{\prime}:=h$. To obtain the inverse transformation (Hp.Dx) to (Hp.Dx.2) let us denote: 
$c_{0}:=a_{0}-a_{1}^{2} / a_{2}, c_{1}:=a_{2}, c_{2}:=-a_{1} / a_{2}, h_{i}{ }^{\prime}:=h_{i}{ }^{\prime}-c_{2}$.

Then:

$$
\begin{aligned}
& c_{0}+c_{1} h_{i} h_{j}=c_{0}+c_{1}\left(h_{i}{ }^{\prime}-c_{2}\right)\left(h_{j}{ }^{\prime}-c_{2}\right) \\
&=a_{0}-a_{1}^{2} / a_{2}+a_{2}\left(h_{i}{ }^{\prime}+a_{1} / a_{2}\right)\left(h_{j}{ }^{\prime}+a_{1} / a_{2}\right) \\
&=a_{0}-a_{1}^{2} / a_{2}+a_{2} h_{i}{ }^{\prime} h_{j}{ }^{\prime}+a_{1}\left(h_{i}{ }^{\prime}+h_{j}{ }^{\prime}\right)+a_{1}^{2} / a_{2} \\
&=a_{0}+a_{1}\left(h_{i}{ }^{\prime}+h_{j}{ }^{\prime}\right)+a_{2} h{ }^{\prime} h_{j}{ }^{\prime} .
\end{aligned}
$$

For any given vector $h^{\prime}$ and coefficients $a_{0}, a_{1}$, and $a_{2}$, expression (Hp.Dx.2) can be written as (Hp.Dx), with $h$, $c_{0}$, and $c_{1}$ given by A2. Now it is enough to show that $(\mathrm{Hp}) \rightarrow$ (Hp.Dx.2). In expressions (Hp.Dx) and (Hp.Dx.2) coefficients $c_{1}$ and $a_{2}$ are nonzero, taking the limit $a_{2} \rightarrow$ 0 and substituting $h_{i}:=a_{0} / 2+a_{1} h_{i}{ }^{\prime}$, we obtain from (Hp.Dx.2) an expression that is infinitely close to (Hp).
2. Now let us assume that we have the solution of (Hp.Dx) for $c_{0}+c_{1} h_{i} h_{j}$. Making substitutions $h_{i}{ }^{\prime}:=c_{0} / 2, c_{0}{ }^{\prime}$ : $=c_{1}$, and $q_{i}{ }^{\prime}:=h_{i}$, we can transform Eq. (3b) to the (Hp.pH) form $h_{i}+h_{j}+c_{0} q_{i} q_{j}$. This proves the relation $(\mathrm{Hp} . \mathrm{Dx}) \rightarrow(\mathrm{Hp} . \mathrm{pH})$.

3. The relation (Hp.pH) $\rightarrow$ (Hp.Dx.pH) is derived similarly as $(\mathrm{Hp}) \rightarrow(\mathrm{Hp} . \mathrm{Dx})$ by proving first that (Hp.Dx.pH) is equivalent to the following formula:

$a_{0}+a_{1}\left(h_{i}{ }^{\prime}+h_{j}{ }^{\prime}\right)+a_{2} h_{i}{ }^{\prime} h_{j}{ }^{\prime}+a_{3}\left(q_{i}{ }^{\prime}+q_{j}{ }^{\prime}\right)+a_{4} q_{i}{ }^{\prime} q_{j}{ }^{\prime}$.

Assuming that $c_{0}:=a_{0}-a_{1}^{2} / a_{2}-a_{3}^{2} / a_{4}, c_{1}:=a_{2}, c_{3}$ : $=a_{4}, c_{2}:=-a_{1} / a_{2}, c_{4}:=-a_{3} / a_{4}, h_{i}:=h_{i}{ }^{\prime}-c_{2}$, and $q_{i}:=q_{i}{ }^{\prime}-c_{4}$, and transforming (Hp.Dx.pH) similarly, as in A1-A3, we obtain A4. In the limit $a_{1} \rightarrow 0, a_{3} \rightarrow 0$, we obtain from A4 an expression that is infinitely close to (Hp.pH). 